\title{
Identification and Clearance Involved in the Formation of Glucuronides of RT-3003, a New Peripheral Blood Flow Enhancer, and Its Metabolite in Rats
}

\author{
Takayuki Kaburagl, ${ }^{a}$ Hiroyuki Yoshitsugu, ${ }^{b}$ Harumi UeKuSA, ${ }^{a}$ Masataka Ishibashi,${ }^{b}$ and \\ Toshio NANBO ${ }^{*, a}$ \\ Institute of Xenobiotic Analyses," 2802-1, Hiratsuka, Shiroi, Inba, Chiba 270-1402, Japan and Research and Develop- \\ mental Department, Takata Seiyaku Co., Ltd., ${ }^{b}$ 203-1 Miyamae, Omiya, Saitama 331-8588, Japan. \\ Received August 13, 1999; accepted October 2, 1999
}

\begin{abstract}
Glucuronides of RT-3003 and its metabolite (9-OH-RT-3003), which was hydroxylated at the 9 position on the benzene ring, were separated by HPLC and identified by liquid chromatography (LC)/MS/MS and NMR. The conjugation sites of these glucuronides were determined by nuclear Overhauser effects (NOE) irradiation; RT-3003 was conjugated at an alcoholic hydroxyl group of the hydroxymethyl moiety, and 9-OH-RT-3003 at a phenolic hydroxyl group on a benzene ring and at an alcoholic hydroxyl group of a hydroxymethyl moiety. On a reversed-phase HPLC of 9-OH-RT-3003, alcoholic glucuronide was eluted later than phenolic glucuronide, indicating the high hydrophobicity of alcoholic glucuronide. Clearance for the glucuronidation $\left(\mathrm{Cl}_{\mathrm{G}}\right)$ of RT-3003 was lower than the summation of $\mathrm{Cl}_{\mathrm{G}}$ for two types of glucuronidation of 9-OH-RT-3003. $\mathrm{Cl}_{\mathrm{G}}$ of 9-OH-RT-3003 was high in phenolic glucuronide. The activity of UDP-glucuronyltransferase (UDPGT) for RT-3003 was 9.63 times that for 9-OH-RT-3003, and the activity ratio of the two types of glucuronidation of 9-OH-RT-3003 was similar to the ratio of the corresponding $C l_{\mathrm{G}}$. The difference between $C l_{\mathrm{G}}$ and UDPGT activity is discussed in association with clearance for the hydroxylation and interaction of substrates with UDPGT.
\end{abstract}

Key words glucuronidation site; nuclear Overhauser effect; liquid chromatography (LC)/MS/MS; clearance

RT-3003,(-) $1 \beta$-Ethyl- $1 \alpha$-hydroxymethyl-1,2,3,4,6,7, 12ba-octahydroindoro-[2,3-a]-quinolizine which was developed by Gedeon Lichter, Ltd. (Hungary) has selective peripheral blood flow enhancing properties in experimental animals. ${ }^{1)}$ The chemical structure of RT-3003 is shown in Fig. 1. This compound has a symmetric center at positions 1 and 12b; RT-3003 is trans-form, referring to the relative position of the $\mathrm{C} 1-\mathrm{C}_{2} \mathrm{H}_{5}$ group and the $\mathrm{C} 12 \mathrm{~b}-\mathrm{H}$ bond. Szombathelyi et $a l .{ }^{1)}$ found that the pharmacological effect of trans-form was higher than that of cis-form.

The structure of RT-3003 suggests various types of glucuronidations: glucuronidation at the hydroxymethyl group, secondary amine, tertial amine and hydroxyl group on the benzene ring formed by monooxidation. The formation rates of these glucuronides are assumed to vary with the types of glucuronides produced by the catalysis of various isoforms of UDP-glucuronyltransferase (UDPGT). Therefore, identification of the structures of glucuronides and pharmacokinetic analyses of glucuronidation are useful in clarifying the elimination of this drug from the body.

The purpose of this study is to identify the various types of glucuronide produced from RT-3003 following the oral administration of ${ }^{14} \mathrm{C}-\mathrm{RT}-3003$ into rats, and to analyze the clearance for glucuronidation associated with the elimination of RT-3003 and its metabolites.

\section{MATERIALS AND METHODS}

Labeled Compound ${ }^{14} \mathrm{C}-\mathrm{RT}-3003$ (specific activity; $14.7 \mathrm{mCi} / \mathrm{mmol}$ ) was obtained from Gedeon Lichter, Ltd. (Hungary). ${ }^{14} \mathrm{C}-9-\mathrm{OH}-\mathrm{RT}-3003$ (specific activity; $9.5 \mathrm{mCi} /$ $\mathrm{mmol}$ ) was obtained by the hydrolysis of biliary excretes: biliary excretes were incubated in excised cecum at $37^{\circ} \mathrm{C}$ for $1 \mathrm{~h}$ and extracted with ethylacetate, followed by purification

* To whom correspondence should be addressed. using HPLC on $300 \times 25 \mathrm{~mm}, \mu$ Bondapak C18 $125 \AA$ (Waters) using solvent $\mathrm{A}, 10 \mathrm{~mm}$ ammonium acetate buffer $(\mathrm{pH}$ 8.0 ) and solvent $\mathrm{B}$, acetonitrile with a linear gradient to $10-$ $30 \% \mathrm{~B}$ in $30 \mathrm{~min}$.

Animal Treatment Male Wistar rats weighing 240$260 \mathrm{~g}$ (8 weeks old) were purchased from Clea Japan, Inc (Tokyo). The bile ducts of all animals were cannulated with polyethylene tubes. To collect urinary samples, vinyl tubes $(4.0 \mathrm{~mm}$ i.d. $\times 4.8 \mathrm{~mm}$ o.d.) were adhered to abdominal skin in a fashion such that the urinary outlets were placed in the tubes.

Purification Procedure Bile was collected for $8 \mathrm{~h}$ following the oral administration of ${ }^{14} \mathrm{C}-\mathrm{RT}-3003$ at a dose of 3 $\mathrm{mg} / \mathrm{kg}$. Bile samples were extracted from Sep-Pak C18 (Waters) with $30 \%$ and $40 \%$ methanol. HPLC of these fractions was performed on an LC-10A system (Shimadzu, Japan) equipped with an LC-10A pump and SPD-10A UV detector. Radioactivity collected with a fraction collector was assayed with a Model 903 liquid scintillation counter (Aloka). The $30 \%$ methanol extracts were first purified by HPLC on $300 \times 25 \mathrm{~mm}, \mu$ Bondapak C18 $125 \AA$ (Waters). The solvent system was as follows: A, $10 \mathrm{~mm}$ ammonium acetate buffer

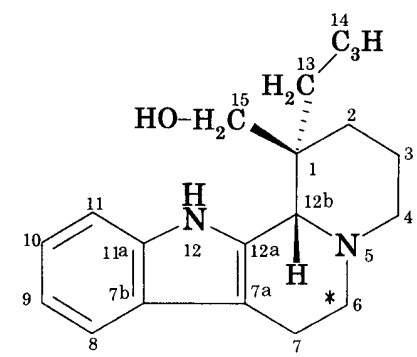

Fig. 1. Chemical Structure of RT-3003

* Position labeled with ${ }^{14} \mathrm{C}$.

(C) 2000 Pharmaceutical Society of Japan 


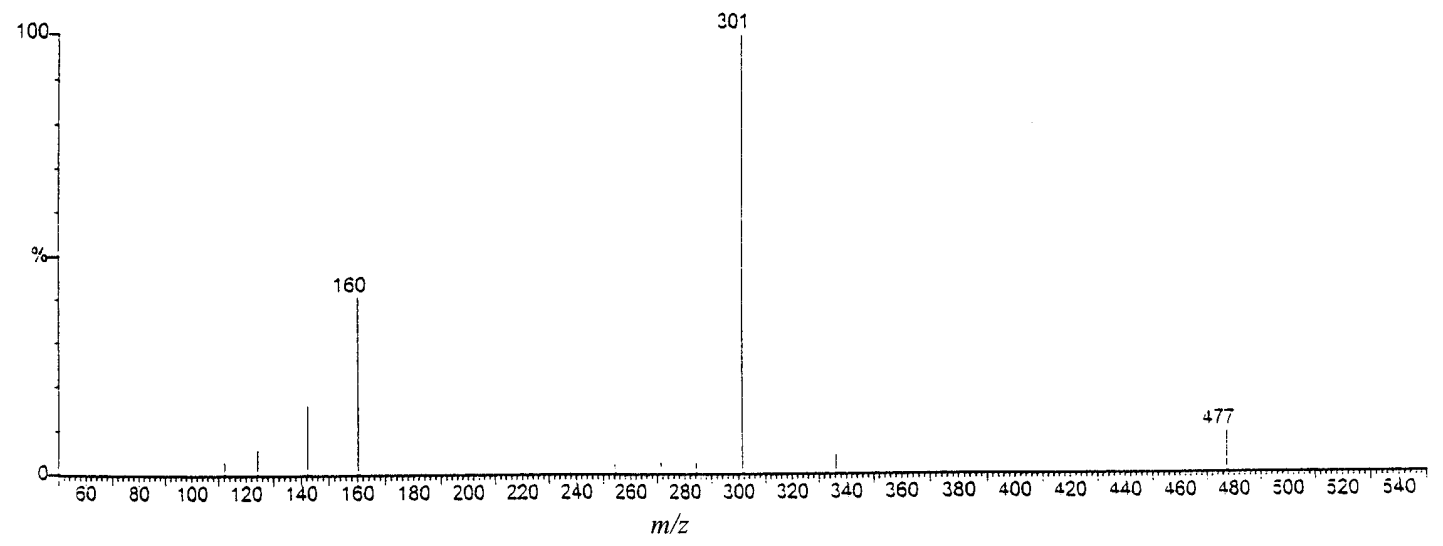

Fig. 2. Product Ion Spectrum of $m / z 477$ of Metabolite M2 Purified from Biliary Excretes

( $\mathrm{pH} 5.0$ ) and $\mathrm{B}$, acetonitrile, with a linear gradient to 0 $30 \% \mathrm{~B}$ in $15 \mathrm{~min}$, followed by a $15 \mathrm{~min}$ hold at $30 \% \mathrm{~B}$ at a flow rate of $1 \mathrm{ml} / \mathrm{min}$. Further purification was achieved by HPLC with $\mu$ Bondapak C18 $10 \mu \mathrm{m}(300 \times 7.8 \mathrm{~mm}$, Waters $)$. The column was eluted with an isocratic phase of A and B $(95: 5)$. Purification of the $40 \%$ methanol extracts was first carried out by using the first HPLC condition for the $30 \%$ methanol extracts. Further purification was performed by HPLC with $\mu$ Porasil $10 \mu \mathrm{m}(300 \times 7.8 \mathrm{~mm}$, Waters $)$. The solvent system was as follows: dichloromethane and methanol containing $0.1 \%$ triethylamine, with a linear gradient to 20 $100 \%$ methanol containing $0.1 \%$ triethylamine in $15 \mathrm{~min}$.

Assay of UDPT Activity The assay of the activity of hepatic microsomal UDPGT was carried out as described previously. ${ }^{2)}$ Briefly, microsomes $(0.15 \mathrm{mg}$ protein) were incubated with $4 \mu \mathrm{mol}$ of UDP-glucuronic acid, $0.4 \mu \mathrm{mol}$ of $\mathrm{MgCl}_{2}$ and $0.5 \mathrm{nmol}$ of ${ }^{14} \mathrm{C}-\mathrm{RT}-3003$ or ${ }^{14} \mathrm{C}-9-\mathrm{OH}-\mathrm{RT}-3003$ in $0.1 \mathrm{ml}$ of $20 \mathrm{~mm}$ Tris- $\mathrm{HCl}$ buffer $(\mathrm{pH} 7.8)$ for $20 \mathrm{~min}$ at $37^{\circ} \mathrm{C}$.

TLC Plates (Merck Silica gel $\mathrm{F}_{254}$ ) were developed in toluene-methanol-triethylamine $(8: 1: 1)$. The areas of radioactivity were assayed. ${ }^{3}$ )

NMR Spectrometry NMR spectra were recorded at 270 MHz using a JEOL EX270 spectrometer (JEOL). Samples were dissolved in $0.5 \mathrm{ml}$ of dimethylsulfoxide- $d_{6}$.

LC/MS/MS Analysis A Micromass tandem quadruple mass spectrometer (Quanttro II, Micromass UK Ltd.) equipped with an electrospray ionization source system, a Hewlett Packard HPLC system (Series 1100, CA, U.S.A.), was employed. HPLC conditions are as follows: TSK-gel ODS-80Ts column $(150 \times 2 \mathrm{~mm})$ was eluted with solvent $\mathrm{A}$, $10 \mathrm{~mm}$ ammonium formate buffer $(\mathrm{pH} 8.0)$ and $\mathrm{B}$, acetonitrile, with a linear gradient to $0-34 \% \mathrm{~B}$ in $30 \mathrm{~min}$.

Calculation of Clearance For the determination of $\mathrm{Cl}_{\mathrm{G}}$ of RT-3003, ${ }^{14} \mathrm{C}-\mathrm{RT}-3003$ was administered orally with $3 \mathrm{mg}$ $(10.6 \mu \mathrm{mol}) / \mathrm{kg}$, and urine and bile were collected for $24 \mathrm{~h}$. The blood samples were taken by cutting carotid arteries at various times until $24 \mathrm{~h}$. To determine the $\mathrm{Cl}_{\mathrm{G}}$ of $9-\mathrm{OH}-\mathrm{RT}$ $3003,{ }^{14} \mathrm{C}-9-\mathrm{OH}-\mathrm{RT}-3003$ was injected intravenously with 3.7 $\mu \mathrm{mol} / \mathrm{kg}$. Biological samples were collected in the same way as ${ }^{14} \mathrm{C}$-RT-3003. Clearance for glucuronidation $\left(\mathrm{Cl}_{\mathrm{G}}\right)$ was determined as follows:

$$
C l_{\mathrm{G}}=\text { amount of glucuronide in the bile and urine } / A U C
$$

where $A U C$ is the area under the curve of the plasma concen- tration of corresponding aglycon; $A U C$ was obtained by adding all the trapezoidal areas from the measured values.

Measurement of Biological Sample The plasma obtained by centrifugation of the blood, urine and bile was extracted from Sep-Pak C18 (Waters) with 50\% methanol. An aliquot of the extracts was injected on HPLC with a column of $\mu$ Bondapak C18 $10 \mu \mathrm{m}(300 \times 7.8 \mathrm{~mm}$, Waters $)$ and eluted with $10 \mathrm{mM}$ ammonium acetate buffer ( $\mathrm{pH} 5.0)$ and acetonitrile $(95: 5)$. The eluate was collected with a fraction collector, followed by the radioactivity assay with a liquid scintillation counter.

\section{RESULTS AND DISCUSSION}

Following the oral administration of ${ }^{14} \mathrm{C}-\mathrm{RT}-3003$ to rats, the excreted radioactivity in bile appeared to be 3.8 times that in urine. A thin layer chromatogram of these excretes showed one radioactive spot on origin, indicating the presence of polar metabolites; thus, these polar metabolites in the bile were purified chromatographically. The metabolites were separated into two fractions by solid phase extraction: fractions extracted with $30 \%$ methanol and with $40 \%$ methanol. On HPLC for the further purification of $30 \%$ methanol extracts, two radioactive peaks appeared at $20 \mathrm{~min}$ (M2) and 35 min (M1). The fractions including these peaks were subjected to analysis by LC/MS/MS and NMR.

M1 and M2 gave a simplified mass spectrum in which an ion of $\mathrm{m} / \mathrm{z} 477$ was observed as a base peak corresponding to the protonated molecular ion $(\mathrm{M}+\mathrm{H})^{+}$of the glucuronide of 9-OH-RT-3003. The product ion spectrum from $(\mathrm{M}+\mathrm{H})^{+}$of $\mathrm{m} / \mathrm{z} 477$ for both metabolites showed a fragment ion of $\mathrm{m} / \mathrm{z}$ 301 which corresponded to the loss of a 176-unit moiety from 477 , a process that is characteristic of glucuronide conjugation (Fig. 2). ${ }^{4)}$ The ion at $m / z \quad 301,16$ mass units higher than RT-3003, further suggested monooxidation to RT-3003.

Figure 3 shows ${ }^{1} \mathrm{H}-{ }^{1} \mathrm{H}$ correlation spectroscopy (COSY) of M1. The aromatic region of M1 shows the presence of three protons and an upfield shift of the signals of two protons of $\mathrm{H}-8(6.66 \mathrm{ppm})$ and $\mathrm{H}-10(6.50 \mathrm{ppm})$ compared with RT-3003 (H-8, 7.00 ppm; H-10, 6.96 ppm), suggesting hydroxylation of the aromatic ring. The characteristics of the anomeric proton $\left(\mathrm{H}-1^{\prime}\right)$ of the glucuronic acid suggested a doublet $\left(J_{1^{\prime}-2^{\prime}}=8.0 \mathrm{~Hz}\right)$ at $4.45 \mathrm{ppm}$. The COSY spectrum showed cross-peaks corresponding to the coupling of the anomeric proton of $\mathrm{H}-1^{\prime}$ to $\mathrm{H}-2^{\prime}$ (3.30 ppm), as well as cou- 

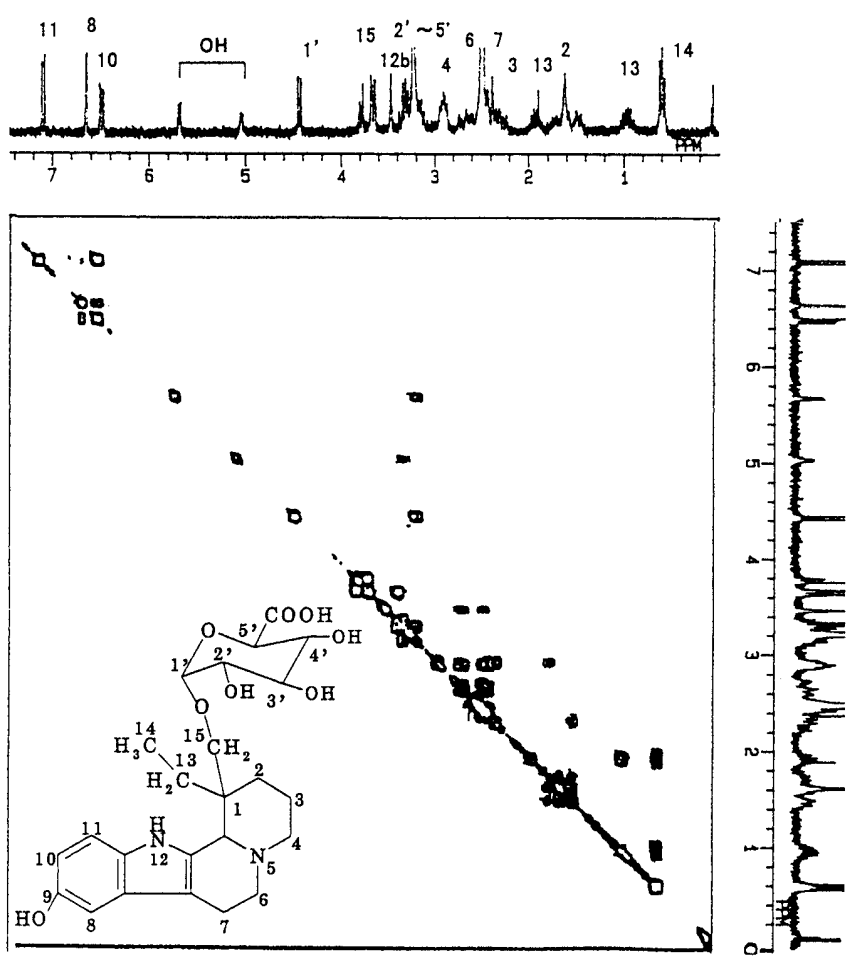

Fig. 3. Two Dimensional ${ }^{1} \mathrm{H}-{ }^{1} \mathrm{H}$ Correlated NMR (COSY) of Metabolite M1 Purified from Biliary Excretes

pling among other protons of the glucuronic acid moiety $(3.2-3.4 \mathrm{ppm})$. Signals of other regions were similar to those of RT-3003, indicating that no metabolic reaction occurred in other regions. The COSY spectrum of M2 resembled that of M1 except that the signals of $\mathrm{H}-8(7.00 \mathrm{ppm})$ and $\mathrm{H}-10(6.78 \mathrm{ppm})$ in the aromatic region exhibited a downfield shift.

From the spectrum of LC/MS/MS and NMR, M1 and M2 were identified as glucuronides of 9-OH-RT-3003. The results that both glucuronides showed differences in their retention time of HPLC and in the chemical shift of protons in the aromatic region suggest that these glucuronides were conjugated at different sites. Thus, the assignment of the site was achieved by nuclear Overhauser effects (NOE) difference spectroscopy (Fig. 4). When a proton of the hydroxymethyl moiety of M1 was irradiated, NOE enhancement was observed at an anomeric proton of the glucuronic acid moiety, indicating that M1 (9-OH-RT-3003-15G) is conjugated at an alcoholic hydroxyl group at the 15 position. Irradiation of the proton attached to C-8 of M2 resulted in NOE enhancement at an anomeric proton of the glucuronic acid moiety, indicating that the glucuronidation site of M2 (9-OH-RT-3003-9G) was the phenolic hydroxyl group at the 9 position on the benzene ring.

On the reversed-phase HPLC, 9-OH-RT-3003-15G was eluted later than 9-OH-RT-3003-9G, indicating that 9-OHRT-3003-15G was more hydrophobic than 9-OH-RT-30039G. Leo et al. ${ }^{5)}$ reported that a chain hydroxyl group contributes greatly to the hydophilicity of a compound comparing with phenolic hydroxyl groups; thus, the late elution of 9-OH-RT-3003-15G would be due partly to the reaction of the alcoholic hydroxyl group with glucuronic acid. To explain the remarkable difference in retention time, however, it is necessary to take into account the possibility that the glu-
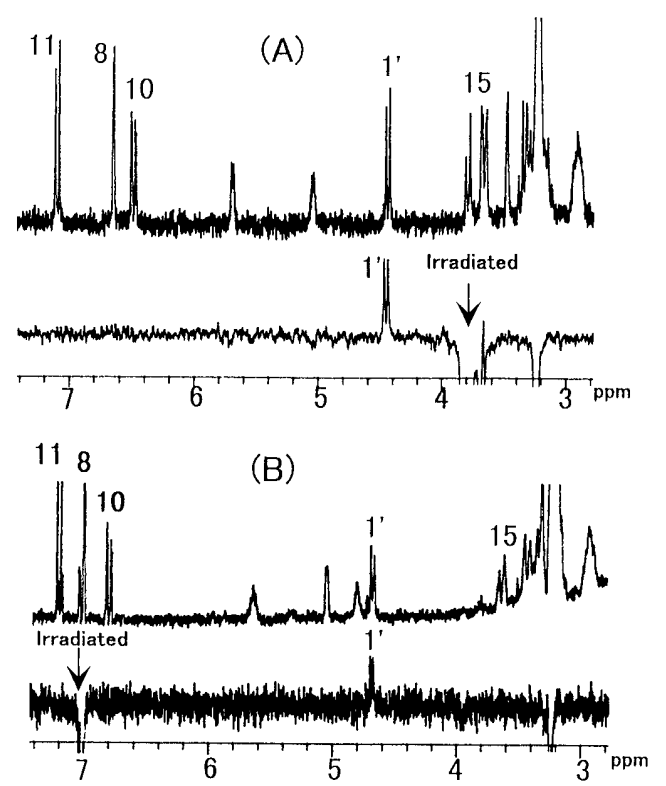

Fig. 4. NOE Difference Spectra of Metabolite M1 (A) and M2 (B)

Table 1. Glucuronides in Bile and Urine after Oral Administration of ${ }^{14} \mathrm{C}$ RT-3003

\begin{tabular}{lcccc}
\hline \hline $\begin{array}{c}\text { Formed } \\
\text { glucuronide }\end{array}$ & $\begin{array}{c}\text { Bile } \\
(\% \text { of dose })\end{array}$ & $\begin{array}{c}\text { Urine } \\
(\% \text { of dose })\end{array}$ & $\begin{array}{c}\text { Total } \\
(\% \text { of dose })\end{array}$ & Bile/Urine \\
\hline RT-3003-15G & $22.1 \pm 3.3$ & $8.1 \pm 1.3^{a)}$ & $30.2 \pm 4.5$ & $2.73 \pm 0.16$ \\
9-OH-RT-3003-9G & $15.3 \pm 2.6^{b)}$ & $5.2 \pm 0.9^{a, b)}$ & $20.5 \pm 3.3^{b)}$ & $2.95 \pm 0.40$ \\
9-OH-RT-3003-15G & $9.9 \pm 1.5$ & $3.3 \pm 0.5^{a)}$ & $13.2 \pm 2.0$ & $3.04 \pm 0.34$
\end{tabular}

Data are the mean \pm S.D. of 5 animals. a) $p<0.05$ compared with the value of bile b) $p<0.05$ compared with the value of $9-\mathrm{OH}-\mathrm{RT}-3003-15 \mathrm{G}$.

curonic acid moiety of 9-OH-RT-3003-15G interacts with other regions of this molecule in a fashion such that the hydrophilicity of this compound decreases.

The MS spectrum of the metabolite (M3) from $40 \%$ methanol extracts also gave a simplified mass spectrum in which the ion of $m / z 461$ corresponding to $(\mathrm{M}+\mathrm{H})^{+}$was observed as a base peak. The product ion from $(\mathrm{M}+\mathrm{H})^{+}$of $m / z$ 461 showed a signal at $m / z 285(\mathrm{M}+\mathrm{H}-176)^{+}$which corresponded to protonated RT-3003 formed by the cleavage of the glucuronic acid moiety. After the hydrolysis of this metabolite with $\beta$-glucuronidase/arylsulfatase, the products were analyzed by TLC, and showed a radioactive spot corresponding to RT-3003. Irradiation of the anomeric proton showed NOE enhancement at a proton at the 15 position. From these results, M3 was identified as a glucuronide of RT3003 (RT-3003-15G), which was conjugated at a hydroxyl group of a hydroxymethyl moiety.

Table 1 shows the ratio of glucuronides in bile and urine after the oral administration of ${ }^{14} \mathrm{C}-\mathrm{RT}$-3003. Three species of glucuronides were mainly excreted into the bile. To examine the effects of the hydroxylation and glucuronidation site on the distribution in both excretion pathways, the urine/bile excretion ratio of glucuronide was calculated; however, no significant difference in the excretion ratio among these glucuronides was observed. The total amount of glucuronide in the excretes was $30.2 \%$ of dose in RT-3003-15G, $20.5 \%$ in 9OH-RT-3003-9G and $13.2 \%$ in 9-OH-RT-3003-15G, showing that glucuronidation played an important role in elimination 
Table 2. Clearance for Glucuronidation and UDPGT Activity of RT-3003 and Its Metabolites

\begin{tabular}{ccc}
\hline $\begin{array}{c}\text { Formed } \\
\text { glucuronide }\end{array}$ & $\begin{array}{c}\text { Clearance } \\
(1 / \mathrm{kg})\end{array}$ & $\begin{array}{c}\text { UDPGT activity } \\
(\mathrm{pmol} / \mathrm{min} \text { mg protein })\end{array}$ \\
\hline RT-3003-15G & $3.53 \pm 0.47^{a)}$ & $91.5 \pm 8.9^{a)}$ \\
9-OH-3003-9G & $3.36 \pm 0.55^{b)}$ & $5.72 \pm 0.61^{b)}$ \\
9-OH-3003-15G & $2.17 \pm 0.31$ & $3.78 \pm 0.42$ \\
& $(5.53 \pm 0.86)^{c)}$ & $(9.50 \pm 0.70)^{c)}$ \\
\hline
\end{tabular}

Data are expressed as mean \pm S.D. of 5 experiments. a) $p<0.05$ compared with the value of the summation of the two types of glucuronide of 9-OH-RT-3003. b) $p<0.05$ compared with the value of 9-OH-RT-3003-15G. c) Summation of the two types of glucuronide of 9-OH-RT-3003.

from the body. Thus, $\mathrm{Cl}_{\mathrm{G}}$ for the formation of these glucuronides was determined. Considering that 9-OH-RT-3003 is produced in the body, the $C l_{\mathrm{G}}$ of $9-\mathrm{OH}-\mathrm{RT}-3003$ was calculated using the data obtained following the intravenous injection of ${ }^{14} \mathrm{C}-9 \mathrm{OH}-\mathrm{RT}-3003$. The dose was designed to be $35 \%$ of the oral dose of ${ }^{14} \mathrm{C}$-RT-3003 from the result that $33.7 \%$ of ${ }^{14} \mathrm{C}-\mathrm{RT}-3003$ appeared to be hydroxylated at the 9 position until $24 \mathrm{~h}$.

As shown in Table $2, \mathrm{Cl}_{\mathrm{G}}$ of RT-3003 was $3.53 \mathrm{l} / \mathrm{kg}, 63.8 \%$ of the summation of $\mathrm{Cl}_{\mathrm{G}}$ of 9-OH-RT-3003. $\mathrm{Cl}_{\mathrm{G}}$ for $9-\mathrm{OH}-$ RT-3003-9G was 1.55 times that for 9-OH-RT-3003-15G. The values of $C l_{\mathrm{G}}$ are assumed to depend on UDPGT activities. Thus, UDPGT activities toward RT-3003 and 9-OH-RT3003 were measured using hepatic microsomes; substrate concentrations were designed to cover the plasma concentrations. The activity toward RT-3003 was 9.63 times the total activity toward 9-OH-RT-3003, showing a high ratio compared with the ratio of the corresponding $\mathrm{Cl}_{\mathrm{G}}$ (Table 2). On the other hand, the UDPGT activity ratio in both types of glucuronidation of 9-OH-RT-3003 was 1.51, similar to the ratio of the corresponding value of $C l_{\mathrm{G}}$. This similarity sug- gests that the in vivo activity of UDPGT is higher in RT-3003 than in 9-OH-RT-3003, and the activity toward RT-3003 is competitively inhibited by the formation of 9-OH-RT-3003 through hydroxylation, resulting in the low value of $C l_{\mathrm{G}}$. To clarify the effect of this hydroxylation, the clearance of the hydroxylation was calculated using the $A U C$ of RT-3003 and the excreted amounts of the glucuronides of 9-OH-RT-3003 instead of its aglycon, since 9-OH-RT-3003 was excreted as glucuronide in the excretes. The clearance obtained was 3.17 $\mathrm{l} / \mathrm{kg}$, which was similar to $\mathrm{Cl}_{\mathrm{G}}$ of RT-3003; therefore, the formation of 9-OH-RT-3003 is assumed to not greatly decrease the glucuronidation. This study could not explain the difference between $C l_{\mathrm{G}}$ and UDPGT activity. To clarify this difference, it would be necessary to examine the interaction between substrates and UDPGT, in association with the physicochemical properties of microsomal membranes and substrates.

Three species of glucuronide in the bile are assumed to be hydrolyzed in the digestive tract, followed by reabsorption into the blood. Reabsorbed RT-3003 would again be glucuronidated with the values of $\mathrm{Cl}_{\mathrm{G}}$ in this study. $\mathrm{Cl}_{\mathrm{G}}$ of reabsorbed 9-OH-RT-3003 would be different from the values which were obtained from the intravenous injection, since these values did not evaluate the first pass effect.

\section{REFERENCES}

1) Szombathelyi Z., Karpati E., Kalaus G., Szabo L., Szantay C., Arzneim.-Forsch., 41, 621-625 (1991).

2) Nanbo T., J. Pharmacobio-Dyn., 5, 849-852 (1982).

3) Nanbo T., J. Pharmacobio-Dyn., 5, 213-221 (1982).

4) Staraub K. M., "Mass Spectrometry in Biomedical Research," ed. by Gaskell S. J., John Wiley \& Sons, Chichester, 1986, pp. 115-134.

5) Leo A., Jow P. Y., Silipo C., Hansh C., J. Med. Chem., 18, 865-868 (1975) 